\title{
Mutation schützt vor Alzheimer-Demenz
}

Fragestellung: Gibt es protektiv wirksame APP-Genvarianten?

Hintergrund: Ein geringer Anteil (1-5\%) aller Alzheimer-Erkrankungen ist strikt genetisch bedingt. Die bislang identifizierten Mutationen betreffen dabei die Gene für das AmyloidVorläufer-Protein (APP) und Komponenten des APP-spaltenden gamma-Sekretase-Komplexes. Über 30 Mutationen in der Protein-kodierenden Region des APP-Gens sind bekannt [1]. Mutationen, die in einem Austausch von Aminosäuren an oder in der Nähe der beta- und gamma-Sekretase-Spaltstelle des APP resultieren, fördern die Entstehung der toxischen A-beta-Peptide oder steigern die Menge an besonders aggregationsfähigem A-beta. Eine Korrelation einer APP-Mutation mit der „Lateonset"-Form der Alzheimer-Demenz wurde bislang nur in einem Einzelfall gefunden (N660Y; [2]); hierbei führte die Mutation zu einem erhöhten Erkrankungsrisiko.

Methodik: APP-Varianten aus dem kodierenden Bereich des APP, ausgehend von kompletten Genomsequenzen von 1795 Personen, wurden analysiert. Mutationen, die in mehr als einem Individuum identifiziert wurden, wurden unter zu Hilfenahme von Chip- und Genealogie-basierten Genotyp Informationen

Jonsson T, Atwal JK, Steinberg

$S$ et al. A mutation in APP

protects against Alzheimer's

disease and age-related cogniti-

ve decline. Nature 2012; 488:

$96-9$ genutzt, um eine Assoziation einzelner Varianten mit der Alzheimer-Demenz zu finden. Für die am stärksten mit einem gesenkten Erkrankungsrisiko korrelierte Variante wurde der Einfluss auf den al- tersbedingten kognitiven Abbau in nicht-dementen Individuen untersucht. Zudem wurde der Einfluss der Mutation auf die APP-Spaltung in Zellkultur untersucht.

Ergebnisse: Das A-Allel des identifizierten SNP (single nucleotide polymorphism rs63750847) führt zu einem Austausch von Alanin gegen Threonin in Position 673 des APP und liegt damit zwei Positionen C-terminal zur beta-Sekretase-Spaltstelle. Es ist häufiger bei Gesunden als bei Alzheimer-Patienten $(0,62 \mathrm{zu}$ $0,13 \%$ ). Beim Vergleich des CPS (Cognitive Performance Score) von 41 Trägern der Variante mit über 3.000 Nicht-Trägern in der Altersklasse der 80 - bis 100-Jährigen zeigte sich in beiden Gruppen ein altersabhängiger Abfall der kognitiven Leistung. Bei Trägern der A673T-Variante war der CPS jedoch durchweg besser als bei Nicht-Trägern. Die Überexpression des mutierten APP in Zellen zeigte eine Reduktion des beta-Sekretase-Spaltprodukts (APPs-beta), während das alternative Spaltprodukt der alpha-Sekretase (APPs-alpha) tendenziell vermehrt gebildet wurde im Vergleich zu Wildtyp-APP. Ebenso wurde die Bildung der amyloidogenen A-beta-Peptide ausgehend von der mutierten Proteinvariante um circa $40 \%$ gesenkt.

Schlussfolgerungen: Mit der Allelvariante A673T des APP konnte das erste Mal eine protektive Mutante im Zusammenhang mit der Alzheimer-Demenz identifiziert werden. Dieser Aminosäureaustausch mildert in gesunden Personen den kognitiven Abbau im Alter. Die Autoren schlagen daher vor, die Alzheimer-Krankheit als Extremfall des altersabhängigen Verlusts kognitiven Potenzials zu betrachten.

\section{-Kommentar von Dr. Kristina Endres}

\section{Nicht der alleinige Auslösefaktor der „Late-onset"-Alzheimer-Demenz}

Die Autoren studierten das Vorkommen der von ihnen beschriebenen seltenen APP-Variante in über 300.000 Skandinaviern. 0,5\% aller Isländer und 0,2-0,5\% aller Finnen, Schweden und Norweger können sich glücklich schätzen, dass sie in ihrem Genom eine Genvariante tragen, die ihnen Schutz vor der Alzheimer-Demenz gewährt. Aus diesen Befunden lässt sich jedoch weit mehr lesen: Transgene Alzheimer-ModellMäuse, die mutiertes menschliches APP überexprimieren, entwickeln zwar oft einzelne Aspekte der Krankheit, zum Beispiel Amyloid-Plaques, aber mit dieser Untersuchung gelang es dezidiert die zentrale Rolle des APP und seiner Spaltprodukte bei der Entstehung der Krankheit im Menschen zu belegen. Zudem bremst diese Mutation anscheinend den normalen Verlust kognitiver Fähigkeiten im Alter aus und zeigt damit eine starke Verbindung zwischen normalen Alterserscheinungen und der Alzheimer-Demenz als Extremum auf. Da die gefundene Variante des APP sehr selten ist, kann es sich hierbei nicht um den alleinigen Faktor handeln, der über die Entstehung der "Late onset"-Alzheimer-Demenz entscheidet. Dennoch bietet die Kenntnis über die seltene Genvariante neue Einblicke in grundlegende Mechanismen der Pathogenese.

\author{
Referenzen \\ 1. Bettens K et al. Hum Mol Genetics 2010; 19: R4-11 \\ 2. Cruchaga C et al. PlosOne 2012; 7: e31039
}

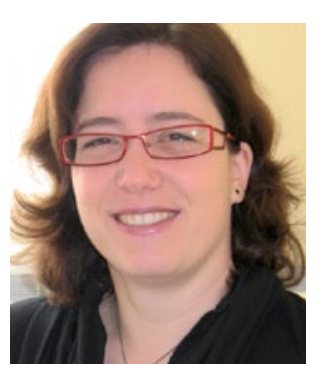

Dr. med. Kristina Endres, Mainz

Klinik für Psychiatrie und Psychotherapie, Universitätsmedizin Johannes Gutenberg-Universität Mainz E-Mail: endres_k@psychiatrie.klinik.unimainz.de 